TITLE:

\title{
Visualization of hydration layers on muscovite mica in aqueous solution by frequency-modulation atomic force microscopy
}

\author{
AUTHOR(S): \\ Kobayashi, Kei; Oyabu, Noriaki; Kimura, Kenjiro; Ido, \\ Shinichiro; Suzuki, Kazuhiro; Imai, Takashi; Tagami, \\ Katsunori; Tsukada, Masaru; Yamada, Hirofumi
}

\section{CITATION:}

Kobayashi, Kei ...[et al]. Visualization of hydration layers on muscovite mica in aqueous solution by frequency-modulation atomic force microscopy. Journal of Chemical Physics 2013, 138(18): 184704.

\section{ISSUE DATE:}

2013-05-09

URL:

http://hdl.handle.net/2433/187961

\section{RIGHT:}

(c) 2013 American Institute of Physics. This article may be downloaded for personal use only. Any other use requires prior permission of the author and the American Institute of Physics. 


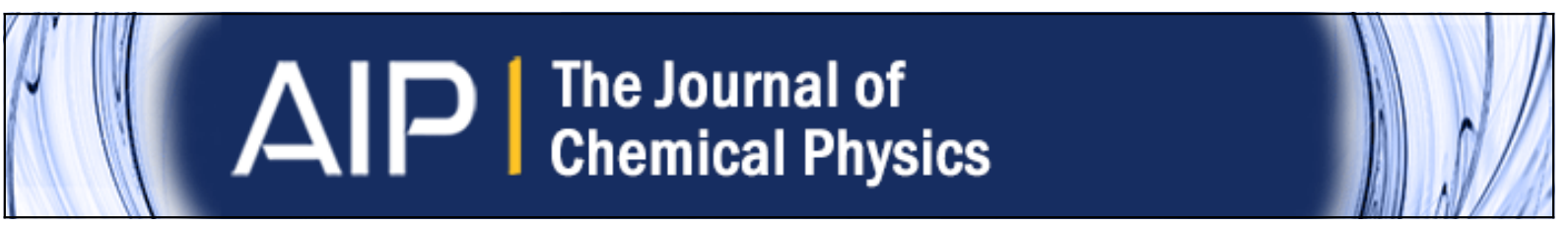

Visualization of hydration layers on muscovite mica in aqueous solution by frequencymodulation atomic force microscopy

Kei Kobayashi, Noriaki Oyabu, Kenjiro Kimura, Shinichiro Ido, Kazuhiro Suzuki, Takashi Imai, Katsunori Tagami, Masaru Tsukada, and Hirofumi Yamada

Citation: The Journal of Chemical Physics 138, 184704 (2013); doi: 10.1063/1.4803742

View online: http://dx.doi.org/10.1063/1.4803742

View Table of Contents: http://scitation.aip.org/content/aip/journal/jcp/138/18?ver=pdfcov

Published by the AIP Publishing

\section{Articles you may be interested in}

A procedure to determine the optimum imaging parameters for atomic/molecular resolution frequency modulation atomic force microscopy

Rev. Sci. Instrum. 81, 093701 (2010); 10.1063/1.3477995

Molecular resolution investigation of tetragonal lysozyme (110) face in liquid by frequency-modulation atomic force microscopy

J. Vac. Sci. Technol. B 28, C4C11 (2010); 10.1116/1.3386383

Three-dimensional force spectroscopy of $\mathrm{KBr}(001)$ by tuning fork-based cryogenic noncontact atomic force microscopy

J. Vac. Sci. Technol. B 28, C4B1 (2010); 10.1116/1.3382230

Frequency noise in frequency modulation atomic force microscopy

Rev. Sci. Instrum. 80, 043708 (2009); 10.1063/1.3120913

Atomically resolved imaging by low-temperature frequency-modulation atomic force microscopy using a quartz length-extension resonator

Rev. Sci. Instrum. 79, 033703 (2008); 10.1063/1.2830937

\section{AlP Re-register for Table of Content Alerts}




\title{
Visualization of hydration layers on muscovite mica in aqueous solution by frequency-modulation atomic force microscopy
}

\author{
Kei Kobayashi, ${ }^{1, a)}$ Noriaki Oyabu, ${ }^{2, b)}$ Kenjiro Kimura, ${ }^{2, c}$ Shinichiro Ido, ${ }^{2}$ Kazuhiro Suzuki, ${ }^{2}$ \\ Takashi Imai, ${ }^{3}$ Katsunori Tagami, ${ }^{4}$ Masaru Tsukada, ${ }^{5}$ and Hirofumi Yamada ${ }^{2}$ \\ ${ }^{1}$ Office of Society-Academia Collaboration for Innovation, Kyoto University, Kyoto 615-8520, Japan \\ ${ }^{2}$ Department of Electronic Science and Engineering, Kyoto University, Kyoto 615-8510, Japan \\ ${ }^{3}$ RIKEN Quantitative Biology Center, Kobe 650-0047, Japan \\ ${ }^{4}$ ASMS Co., Ltd., Tokyo 141-0022, Japan \\ ${ }^{5}$ WPI-Advanced Institute for Materials Research, Tohoku University, Sendai 980-8577, Japan
}

(Received 10 February 2013; accepted 19 April 2013; published online 9 May 2013)

\begin{abstract}
A three-dimensional interaction force mapping experiment was carried out on a muscovite mica surface in an aqueous solution using a high-resolution and low-thermal drift frequency-modulation atomic force microscope. By collecting oscillatory frequency shift versus distance curves at the mica/solution interface, complicated hydration structures on the mica surface were visualized. Reconstructed two-dimensional frequency shift maps showed dot-like or honeycomb-like patterns at different tip-sample distances with a separation of $0.2 \mathrm{~nm}$ with each other, which agree well to the water molecule density maps predicted by a statistical-mechanical theory. Moreover, site-specific force versus distance curves showed a good agreement with theoretically calculated site-specific force curves by a molecular dynamics simulation. It is found that the first and second hydration layers give honeycomb-like and dot-like patterns in the two-dimensional frequency shift images, respectively, corresponding to the lateral distribution function in each layer. (c) 2013 AIP Publishing LLC. [http://dx.doi.org/10.1063/1.4803742]
\end{abstract}

\section{INTRODUCTION}

Solid-liquid interfaces play important roles in a wide variety of physical, chemical, and biological processes. ${ }^{1-4}$ Investigations of atomic-scale structures and interactions at the solid-liquid interfaces are essentially important for understanding these processes. We recently achieved atomicresolution imaging of a muscovite mica surface in water ${ }^{5}$ by frequency-modulation atomic force microscopy (FMAFM) ${ }^{6-8}$ a technique that has been mainly used in vacuum environments, and made it possible to explore solid-liquid interface properties with atomic resolution. We previously reported FM-AFM images of the mica surface with a dot-like pattern and a honeycomb-like pattern. ${ }^{5}$ Moreover, we showed a frequency shift versus tip-sample distance curve measured at the mica-water interface in the paper, suggesting the existence of the hydration layers.

The hydration structures on the solid surfaces have been extensively studied by the surface scientists for decades, ${ }^{9}$ and the importance of the water molecules on the structural stability and function of the biological molecules has long been recognized in the field of molecular biology. ${ }^{10}$ Structures of the hydration layers as a function of the distance from the surfaces have been studied by AFM experiments. ${ }^{5,11-14}$ However, since the hydration layers are not always uniform on

\footnotetext{
a)E-mail: keicoba@iic.kyoto-u.ac.jp. Present address: The Hakubi Center for Advanced Research and Department of Electronic Science and Engineering, Kyoto University, Kyoto 615-8510, Japan.

b) Present address: National Instruments Japan Corporation, Shibadaimon, Minato-ku, Tokyo 105-0012, Japan.

c) Present address: Department of Chemistry, Kobe University, Kobe 657-8501, Japan.
}

the solid surfaces and molecular surfaces, it has been imperative to study the structures of the hydration layers at atomic/molecular resolution. We recently demonstrated visualization of structured water layers by performing twodimensional (2D) frequency shift mapping by FM-AFM on a mica surface and a graphite surface, and on bacteriorhodopsin proteins in a purple membrane. ${ }^{15,16}$ However, precise comparison of the experimental data with theoretically calculated hydration structures or hydration forces ${ }^{17-20}$ requires threedimensional (3D) experimental data, which allows us to identify the relationship between the atomic and molecular species on the surfaces and their specific hydration structures.

There have been several difficulties for reproducible 3D frequency shift mapping in liquids such as a large, linear, and nonlinear thermal drift of the tip position relative to the surface. Recent technical developments in FM-AFM made it possible to perform 3D force mapping experiments at the solidliquid interfaces. ${ }^{21,22}$ Here we show 3D frequency shift map at the mica-water interface, and compare the 3D frequency shift map with a theoretically calculated 3D distribution of the water molecules. Moreover, we compare the force versus distance curves at various tip positions map with the theoretical curves.

\section{EXPERIMENTAL SETUP AND THEORETICAL METHODS}

We used an FM-AFM instrument working in liquids that we developed based on a commercial AFM (SPM-9600, Shimadzu), with home-built electronics ${ }^{23}$ and a home-built controller using a field-programmable gate array module pro- 
grammed with LabVIEW (National Instruments). We modified the optical beam displacement sensor of the original AFM instrument in the same way as described in Ref. 24. The AFM instrument was placed in a temperature-regulated enclosure (CN-40A, Mitsubishi Electric Engineering). The temperature inside the enclosure was kept at $20{ }^{\circ} \mathrm{C}$ with a fluctuation of $\pm 0.1{ }^{\circ} \mathrm{C}$. The lateral and vertical thermal drift in liquid was typically less than $1 \mathrm{~nm} / \mathrm{min}$ in liquids. A muscovite mica plate (Ruby Clear, Furuuchi Chemical) was cleaved and a droplet of $1 \mathrm{~mol} / \mathrm{l} \mathrm{KCl}$ solution was placed on the mica. A highly doped Si cantilever with a gold coating on the backside (PPP-NCHAuD, Nanosensors), whose nominal spring constant and resonance frequency in water were $40 \mathrm{~N} / \mathrm{m}$ and $153 \mathrm{kHz}$, was used. The liquid cell was sealed to prevent evaporation. The oscillation amplitude was kept at $0.24 \mathrm{~nm}$ peakto-peak.

It should be noted that we performed the measurement in a $\mathrm{KCl}$ solution as we often add salts in an imaging solution for FM-AFM in liquids. The lateral resolution in the topographic imaging and the reproducibility in the hydration force measurements are generally improved in strong electrolyte solutions because of the suppression of a long-range double layer force. As is also the case of the mica-electrolyte interface, the surface charges on the mica are compensated by the electric double layer in electrolyte solutions. We actually performed the same experiments in pure water and in $\mathrm{KCl}$ solutions of various concentrations, and we found the difference in the long-range double layer force, but no significant difference for the oscillatory hydration force that we focused in this study. $\mathrm{K}^{+}$ions are not taken into account in the simulations described later in this paper, also because the number of $\mathrm{K}^{+}$ ions in the volume of concern (about $10 \mathrm{~nm}^{3}$ ) is utmost 5 at a high salt concentration of $1 \mathrm{~mol} / \mathrm{l} \mathrm{KCl}$ and they may not be regularly arranged on the mica surface.

Procedures of 3D frequency shift mapping in our AFM system are schematically shown in Fig. 1. The tip was engaged to the sample surface, and the tip-sample distance was controlled by keeping the frequency shift constant. A slow ramp signal was added to $z$-piezo for correction of the linear vertical drift throughout the measurement. ${ }^{25}$ After the tip- sample distance feedback control was disabled, 2D frequency shift maps in $z-x$ planes which are slightly separated in $y$ direction were collected as shown in Fig. 1(a). Each 2D frequency map ( $z-x$ slice) was collected in a $z-x$ plane with dimensions of $3 \mathrm{~nm}$ ( 256 pixels) in $z$ direction by $3 \mathrm{~nm}$ (128 pixels) in $x$ direction. At each $x$ position, the frequency shift versus distance curve was recorded while the tip was approached to the sample surface with a tip velocity of $450 \mathrm{~nm} / \mathrm{s}$ by applying a ramp signal of $75 \mathrm{~Hz}$. The ramp signal was immediately stopped when the frequency shift signal reached a predetermined threshold value, $2250 \mathrm{~Hz}$ in this case, and the tip was retracted to the original position as shown in Figs. 1(b) and 1(c). The threshold value of $2250 \mathrm{~Hz}$ corresponded to the interaction force of about $0.4 \mathrm{nN}$. This threshold value was determined at the maximum in the range where the frequency shift curves could be reproducibly collected; if the threshold value was set at a larger value than this, the measurements were not reliable because of unstable cantilever oscillation or tip change.

By employing the tip retraction function, 67 slices of 2D frequency shift maps were safely obtained in $168 \mathrm{~s}$, including data writing time. The dimensions of 3D frequency shift map were $3 \mathrm{~nm} \times 3 \mathrm{~nm} \times 2.3 \mathrm{~nm}$. After we obtained the 3D data set, we corrected nonlinear drift in $z$ direction as follows. First, we averaged all the frequency shift curves in $x$ direction for each $z-x$ slice. Since each $z-x$ slice almost equally contains the frequency shift curves measured on the various atomic sites on the mica surface, all averaged curves show almost the same oscillatory features at the same tip-sample distance. The common oscillatory feature allowed us to calculate the drift offset of the tip position in $z$ direction as a function of $y$ position and fit the function with a polynomial function. Then all $z-x$ slices were aligned using the polynomial function so that the common oscillatory features in the averaged curves appear at the same height. Movie files (available as the supplementary material ${ }^{32}$ ) were created using the corrected 3D frequency shift data using MicroAVS (Cybernet Systems Co. Ltd.).

We compare the 3D frequency shift data and force data to theoretical 3D data of atomic distribution of water molecules

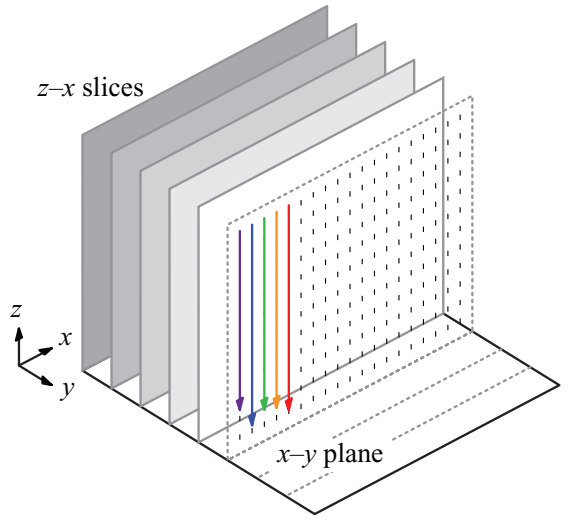

(a)

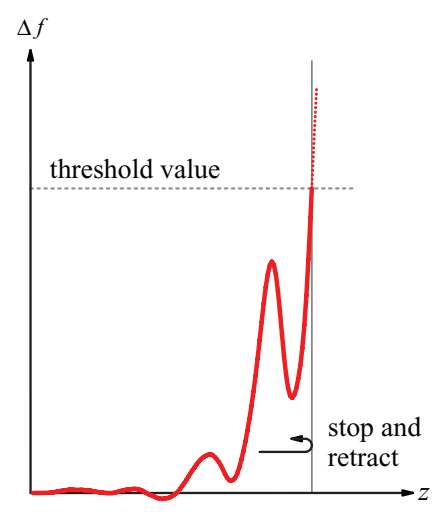

(b)
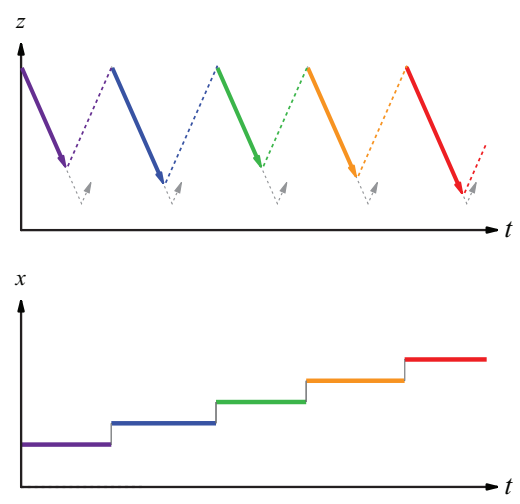

(c)

FIG. 1. Schematics of procedures of 3D frequency shift mapping. (a) 3D frequency shift data were collected by performing 2D frequency shift mapping in $z-x$ planes, which are slightly separated in $y$ direction. (b) In each frequency shift versus distance curve measurement, the tip was retracted immediately if the frequency shift signal reached to the threshold value to avoid the tip crash. (c) Timing charts showing tip movements in $z$ and $x$ directions during $2 \mathrm{D}$ frequency shift mapping. 


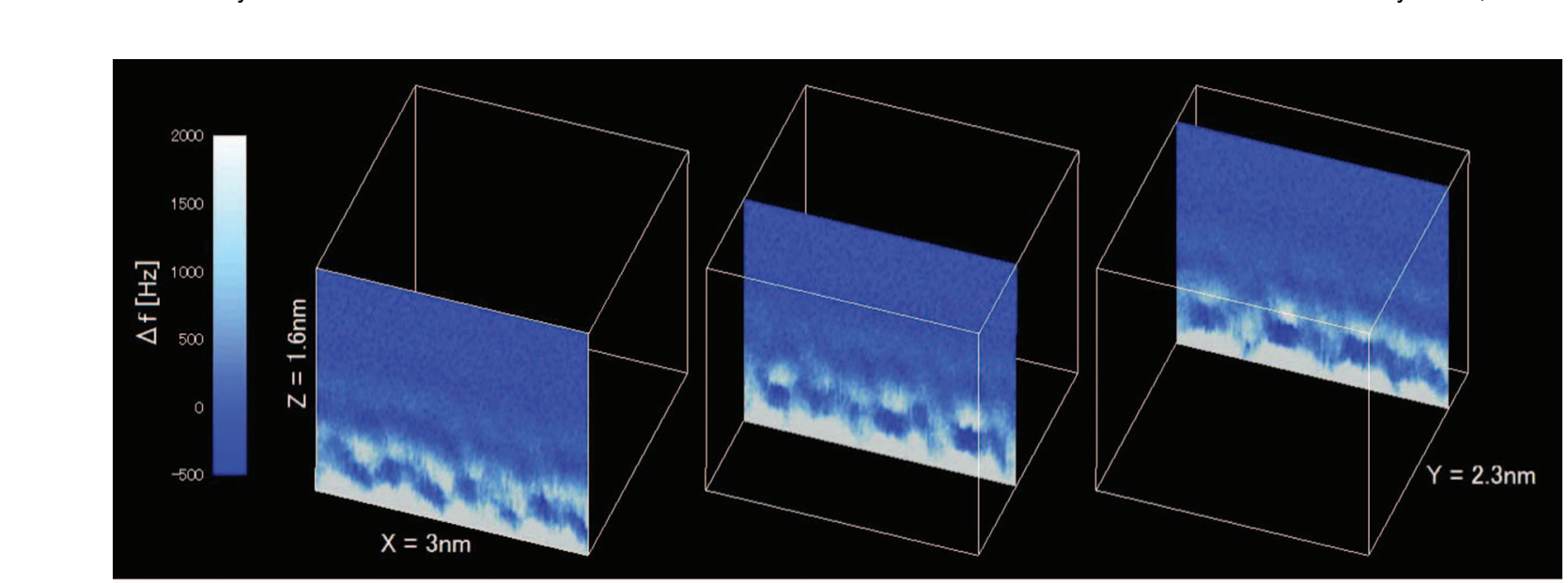

J. Chem. Phys. 138, $184704(2013)$

(a)

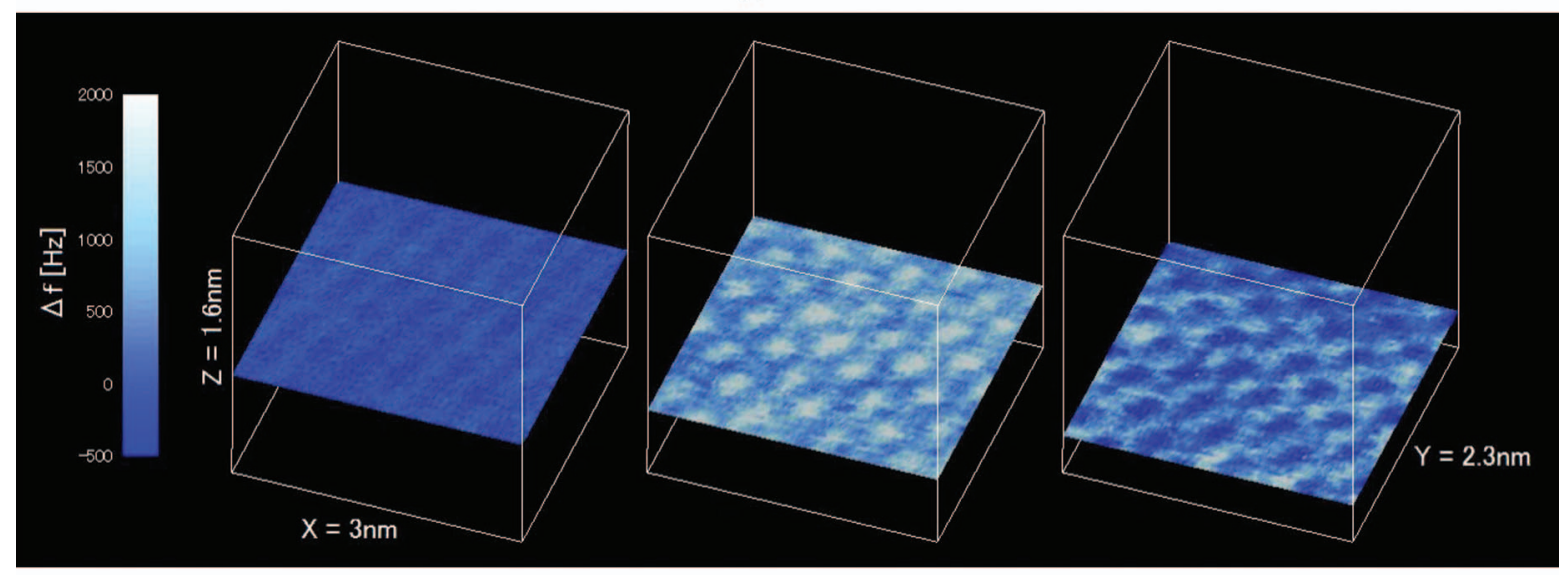

(b)

FIG. 2. (a) Snapshots from a movie of 3D frequency shift data sequentially showing collected $z-x$ slices. (b) Snapshots from a movie showing reconstructed $x-y$ slices as a function of the distance from the surface.

by a statistical-mechanical theory of solvation, known as $3 \mathrm{D}$ reference interaction site model (RISM) theory, and theoretical 3D tip-sample interaction force data calculated by the molecular dynamics (MD) method. Details of the 3D-RISM method and the references are given in Ref. 15. The atomic distribution of water molecules was calculated for a muscovite mica model in pure water at $300 \mathrm{~K}$. Note that the existence of the tip was not taken into account in the 3D-RISM calculation. On the other hand, the MD simulation, as published in Ref. 18, gives 3D distribution of tip-sample interaction force. The interaction force between a tip, modeled by a $(10,0)$ single-wall carbon nanotube with capped ends, and a muscovite mica in pure water at $300 \mathrm{~K}$ was calculated.

\section{RESULTS AND DISCUSSION}

Figure 2 shows snapshots from movie files (available as the supplementary material ${ }^{32}$ ) of the $3 \mathrm{D}$ frequency shift map. The $z$-range of the volume of the shown data is $1.6 \mathrm{~nm}$ after trimming away the volumes without meaningful data. Figure 2(a) shows three $z-x$ slices, as originally obtained in the experiments. The $z-x$ slices show the complicated contrasts reflecting $2 \mathrm{D}$ hydration structures in each $z-x$ plane. One of the movies of the supplementary material ${ }^{32}$ sequentially shows all $z-x$ slices in the manner they were collected, which visualizes how the hydration structures differ in in- dividual $z-x$ slices. Figure 2(b) also shows three $x-y$ slices, reconstructed from the $3 \mathrm{D}$ frequency shift data, which show variations of the frequency shift in $x-y$ planes whose distances from the sample surface are different. In the left slice image, faint contrasts of stripes are recognized, which might be attributed to the anisotropy of the tip shape considering the sixfold symmetry of the mica surface. The center slice image shows 2D frequency shift variation in $x-y$ plane when the tip was approached more to the surface, where a dot-like pattern was visualized. When the tip was further approached, the patterns turned into a honeycomb-like pattern, as shown in the right slice image. Another movie of the supplementary material $^{32}$ sequentially shows reconstructed $x-y$ slices in the direction of the tip approach from the far distance, which visualizes how the dot-like pattern evolves into the honeycomblike pattern.

The middle and right $z-x$ slices shown in Fig. 2(b) with the dot-like and honeycomb-like patterns were represented in Figs. 3(a) and 3(b), respectively. The lateral thermal drift was corrected by shearing the images. The lateral drift rate was estimated to be about $0.3 \mathrm{~nm} / \mathrm{min}$. These images, which are equivalent to the constant height images obtained by the conventional FM-AFM experiments, represent the variations of the frequency shift in $x-y$ planes with the most prominent dot-like and honeycomb-like patterns, both with a periodicity of $0.52 \mathrm{~nm}$, as later depicted in Fig. 3(d). The separation 


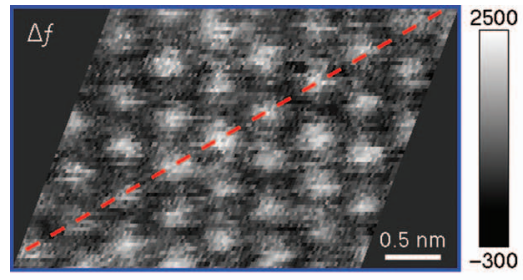

(a)

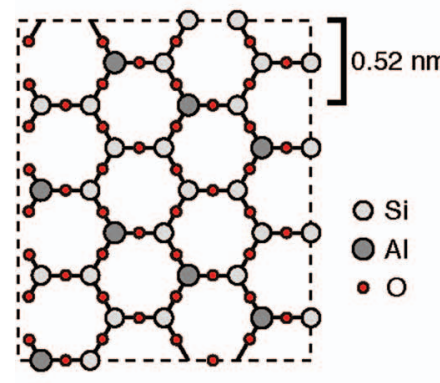

(d)

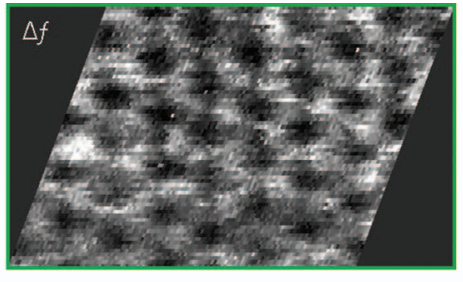

(b)

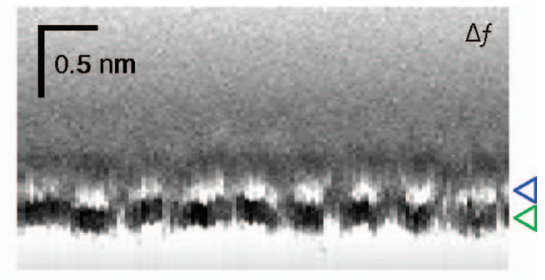

(c)

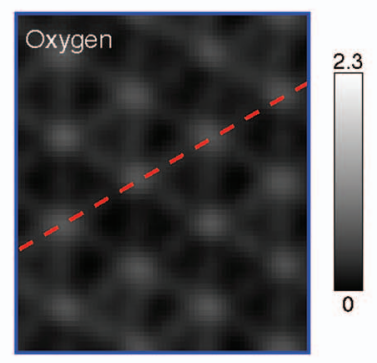

(e)

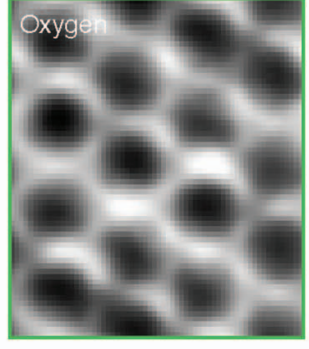

(f)

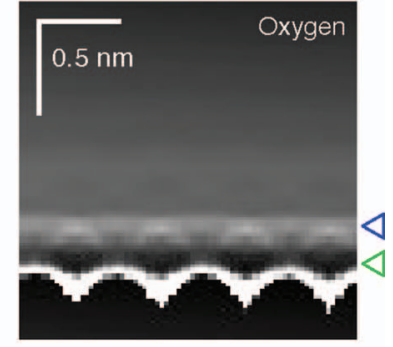

(g)

FIG. 3. (a) and (b) 2D frequency shift maps ( $z-x$ slices) showing dot-like and honeycomb-like patterns. The honeycomb-like pattern was visualized when the tip was approached further by $0.2 \mathrm{~nm}$ to the surface from the tip-sample distance with the dot-like pattern. (c) $2 \mathrm{D}$ frequency shift map in the $z-x$ plane including the dotted line in (a). The triangles on the right of the map represent the locations of the two $x-y$ slices in (a) and (b). (d) Schematic of surface atoms of the muscovite mica model used in the 3D-RISM calculation. (e), (f) Calculated 2D oxygen density maps showing dot-like and honeycomb-like features. (g) Calculated 2D oxygen density map in the $z-x$ plane including the dotted line in (e). The triangles on the right of the map represent the locations of the two $x-y$ slices in (e) and (f).

of the two $x-y$ slices in $z$ direction was $0.2 \mathrm{~nm}$. Figure 3(c) shows a $2 \mathrm{D}$ frequency shift map in the $z-x$ plane including the dotted line in Fig. 3(a), which crosses the bright dots of the dot-like pattern. The locations of the two $x-y$ slices in

(a)

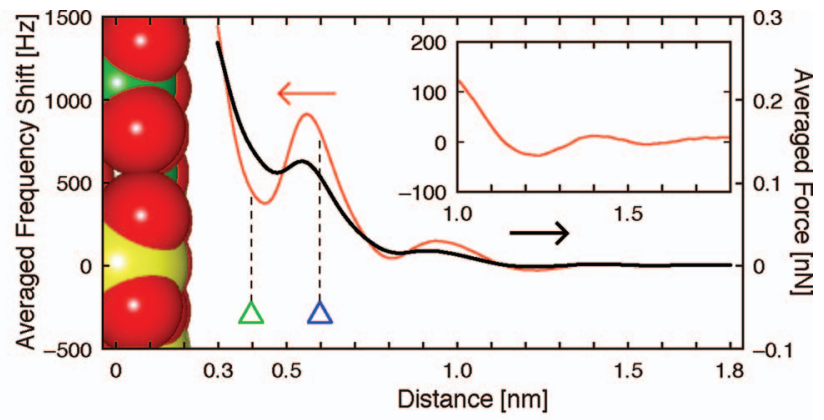

(b)

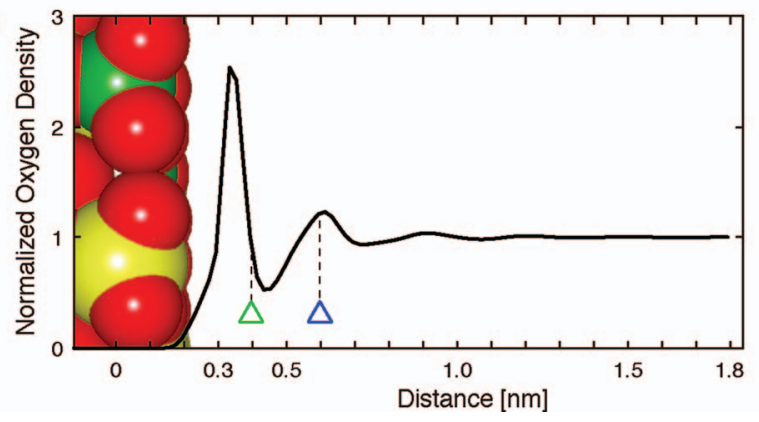

FIG. 4. (a) Averaged frequency shift versus distance curve and averaged force versus distance curve on mica surface in $\mathrm{KCl}$ aqueous solution. Inset shows a magnified frequency shift curve to clearly show the oscillatory features. The triangles represent the locations of the two $x-y$ slices in Figs. 3(a) and 3(b). (b) Normalized oxygen density profile derived by averaging 3D oxygen density map obtained by the 3D-RISM calculation. The triangles represent the locations of the two $x-y$ slices in Figs. 3(e) and 3(f).
Figs. 3(a) and 3(b) are indicated by the triangles. In this figure, it becomes clear that the dark contrasts are beneath the bright dots, namely, the inversion of the contrast between Figs. 3(a) and 3(b).

We first compare the experimental 3D frequency shift data with the calculated 3D distribution of water molecules by the 3D-RISM method. The surface of the mica model used in the 3D-RISM calculation is shown in Fig. 3(d). By creating movie files of the 3D oxygen density distribution in the same way as the experimental frequency shift data, interestingly, we found the oxygen density also shows similar dot-like and honeycomb-like patterns with a separation of about $0.2 \mathrm{~nm}$ with each other. The honeycomb-like pattern was found at the distance closer to the mica surface. Two $x-y$ slices with a separation of $0.2 \mathrm{~nm}$ in $z$ direction were reconstructed from the 3D oxygen density data, and shown as Figs. 3(e) and 3(f). The two $x-y$ slice images show variation of the oxygen density, which is equivalent to the water molecule density, in $x-y$ planes whose distances from the sample surface are different. Note that the oxygen density presented here was normalized so that it becomes 1 at the far distance. The honeycomb-like pattern follows locations of the surface atoms, while the dots in the dot-like pattern are on the hollow sites. Figure $3(\mathrm{~g})$ shows a $2 \mathrm{D}$ oxygen density map in the $z-x$ plane including the dotted line in Fig. 3(e), which crosses the bright dots of the dot-like pattern. The locations of the two $x-y$ slices in Figs. 3(e) and 3(f) are indicated by the triangles in the same way as the experimental result. Here the inversion of the contrast between Figs. 3(e) and 3(f) were again shown.

We defined the origin of $z$-axis in the 3D frequency shift data so that the $z$-distances of the dot-like and honeycomb-like patterns in both the frequency shift data and the oxygen den- 
sity data match, which are separated by $0.2 \mathrm{~nm}$ in both data. Figure 4(a) shows an averaged frequency shift versus distance curve for all pixels in $x-y$ plane. The origin of $z$-axis is defined at the averaged location of outermost $\mathrm{Si}$ and $\mathrm{Al}$ atoms in the mica model used in the 3D-RISM calculation, which is also shown in the figure using a visualization software VESTA. ${ }^{26}$ $\mathrm{Si}, \mathrm{Al}$, and $\mathrm{O}$ atoms were drawn with radii of $0.2 \mathrm{~nm}$, $0.17 \mathrm{~nm}$, and $0.14 \mathrm{~nm}$, respectively. The frequency shift data below $z=0.3 \mathrm{~nm}$ were not available in some $x-y$ pixels because of the tip retraction, while the outermost surface of the mica could extend to $z=0.2 \mathrm{~nm}$ as shown by the mica model, if the alignment of the $z$-axis is correct. It should be noted here that it is desirable to calibrate the $z$ coordinate against the mica surface, however it is not an easy task. In the FMAFM measurements, we simultaneously record the damping of the cantilever as well as the frequency shift. When the tip starts to directly interact the mica surface, we observe a sudden onset of a strong damping of the cantilever, as we recently reported. ${ }^{27}$ This indication allows us to determine the $z$ coordinate but with a precision of a few angstroms.

There are three distinct maxima in the averaged frequency shift curve, at $z=0.5-0.6 \mathrm{~nm}, z=0.9-1.0 \mathrm{~nm}$, and $z=1.4 \mathrm{~nm}$. The triangles in the plot indicate the locations at which the dot-like and honeycomb-like patterns were obtained. Note that we reported the dot-like and honeycomb-like patterns in our previous paper, ${ }^{5}$ as mentioned above. However, the image pattern in our previous paper had not necessarily been a function of the tip-sample distance, but the pattern sometimes changed with an indication of the tip change under the same frequency setpoint. Also taking into account that the dot-like pattern in Ref. 5 was very clear unlike the dot-like pattern observed in this study, we consider that the dot-like pattern in Ref. 5 was not representing the lateral distribution of the water molecules, but the tip apex was scanned laterally probably under the direct interaction with the mica surface, which occasionally brought the clear dot-like lattice pattern.

The averaged frequency shift curve was converted to an averaged force versus distance curve using a method by Sader and Jarvis, ${ }^{28}$ which was also plotted in the figure. The oscillatory nature was still maintained in the averaged force curve, although the maxima are not prominent. We show an averaged oxygen density profile as a function of the distance from the surface atoms in Fig. 4(b). The profile was obtained by averaging the oxygen density distribution, obtained by the 3DRISM calculation, in $x-y$ plane. The oxygen density is equivalent to the water molecule density, and it is normalized to its bulk density. The first hydration layer, the density peak at $z$ $=0.3 \mathrm{~nm}$ in the averaged profile in Fig. 4(b), is consistent with those previously measured by X-ray crystal truncated $\operatorname{rod}(\mathrm{CTR})$ measurement ${ }^{29}$ and theoretical simulations. ${ }^{18,30,31}$ Note that the existence of a water molecule in a ditrigonal cavity (the adsorbate layer) was also reported in Refs. 29-31. The adsorbate layer was also reproduced by the 3D-RISM calculation, as well as by the MD simulation, ${ }^{18}$ but it is not visible in the averaged profile in Fig. 4(b). The locations of the maxima and minima in Fig. 4(b) well correspond to those in Fig. 4(a), namely, the maxima in the frequency shift or force correspond to the hydration layers, in which the water densities are greater than the bulk density.

We also converted individual frequency shift versus distance curves at all pixels in $x-y$ plane to compare site-specific interaction forces to the theoretical 3D force data by the MD calculation. Figures 5(a) and 5(b) are the $x-y$ slices reconstructed from the $3 \mathrm{D}$ force data, at the tip-sample separations same as those in Figs. 3(a) and 3(b). The dot-like features are still prominent, however the honeycomb-like patterns are no longer prominent, it rather shows a vertical stripe pattern, probably again due to the tip anisotropy. Figure 5(c) shows a $2 \mathrm{D}$ force map in the $z-x$ plane including the dotted line in

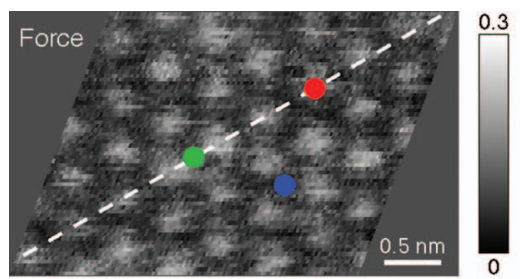

(a)

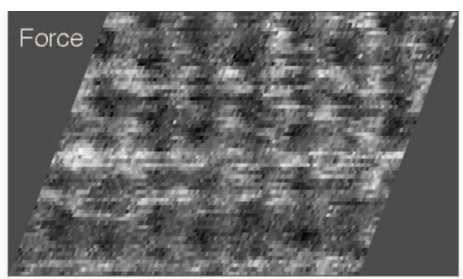

(b)

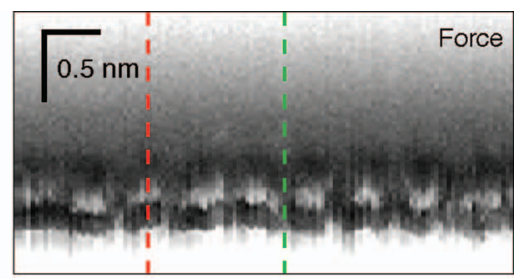

(c)

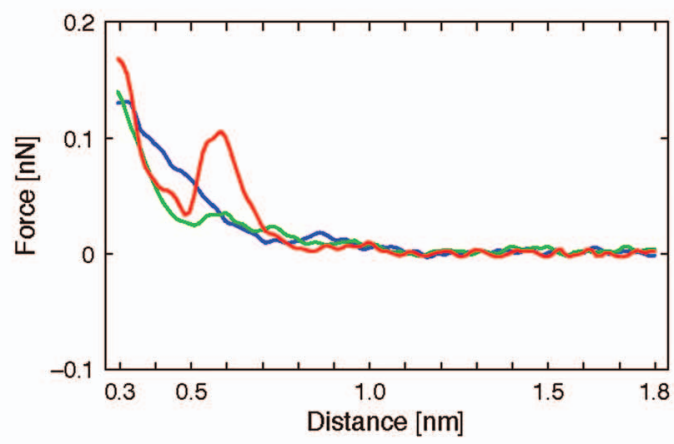

(d)

FIG. 5. (a) and (b) 2D force maps ( $z-x$ slices) at the same tip-sample separations of Figs. 3(a) and 3(b), reconstructed from the 3D force data. (c) 2D force map in the $z-x$ plane including the dotted line in (a). (d) Site-specific interaction force versus distance curves on the locations indicated by the dots in (a). 
Fig. 5(a), which crosses the bright dots of the dot-like pattern. The interaction force versus distance curve on the dot, which corresponds to the honeycomb core of the mica surface, and that between the dots corresponding to the bridge site, were extracted from the 2D force map as indicated in the map, and they were plotted in Fig. 5(d). The interaction force on a center of three adjacent dots corresponding to $\mathrm{Si}$ or $\mathrm{Al}$ site, indicated as a dot off the line in Fig. 5(a), was also plotted. The $z$-range and colors of the curves were the same as those of Fig. 2 in Ref. 18. All the curves in Fig. 5(d) are qualitatively similar to those by the MD simulation, except for the magnitude of the interaction force. For example, the peak position in the experimental curve on the dot of the dot-like pattern agrees well with the theoretical curve on the honeycomb core of the mica surface.

From the comparisons of the experimental data with the 3D-RISM and MD calculations, we are now sure that the definition of the origin of $z$-axis as depicted in Fig. 4 is correct. The frequency shift maximum in the averaged frequency shift curve at $z=0.5-0.6 \mathrm{~nm}$ corresponds to the hydration layer in the 3D-RISM data, which was also reported by the X-ray CTR measurement and referred to as the second hydration layer. ${ }^{29}$ As the dot-like pattern was obtained at the tip distance very close at the frequency shift maximum, the dot-like pattern in Figs. 3(a) and 3(e) reflects the lateral structures of the second hydration layer. On the other hand, the honeycomb-like pattern appears in Figs. 3(b) and 3(f) reflects the lateral structures of the first layer. The brightest areas in the 2D oxygen density map calculated by the 3D-RISM shown in Fig. 3(g) correspond to the first layer, as defined in Ref. 29. We consider that it would be difficult to visualize the adsorbate layer by FM-AFM because the tip should pick the ditrigonal cavity and displace the water molecule inside, which is not probable with a tip of a radius on the order of nanometers, as simulated by the MD calculation in Ref. 18 .

Finally, it should be noted that the experimentally measured force was about one order of magnitude smaller than the theoretically calculated interaction force by the MD calculation probably because the tip model was a carbon nanotube tip, while the real tip was presumably a silicon dioxide tip with a nanometer-scale asperity. The carbon nanotube is one of the promising candidates for a well-defined tip, while the silicon diode tip is not only poorly defined but it is known to be easily contaminated by the surface materials. A series of the same experiments using a carbon nanotube tip is currently ongoing to further justify the comparison.

\section{CONCLUSIONS}

We collected a 3D frequency shift map on a mica surface in an aqueous solution of $\mathrm{KCl}$ using a high-resolution and low-thermal-drift FM-AFM system. The 2D frequency shift maps reconstructed from the 3D data showed a dot-like pattern corresponding to the lateral structures of the second hydration layer, which turned to a honeycomb-like pattern reflecting the first hydration layer when the tip was approached further to the surface by $0.2 \mathrm{~nm}$. It was also shown that the site-specific interaction force curves showed excellent agreements with the interaction forces on the honeycomb core site, bridge site, and $\mathrm{Si} / \mathrm{Al}$ site, respectively, predicted by the MD calculation. It is found from the comparisons of the experimental data to the simulation and theoretical calculations that the first hydration layer and the second hydration layer give honeycomb-like and dot-like patterns in the 2D frequency shift images obtained at the mica-water interface, corresponding to the lateral distribution function in each layer. However, for further discussion on the interpretation of the image contrasts obtained by the FM-AFM experiments, FM-AFM measurements using a well-defined tip and theoretical calculations using a realistic tip model are demanded. Finally, we would like to note here that we could not change the imaging solution without changing the tip conditions or the imaging area with the current experimental setup, but it is interesting to perform the same experiments while exchanging the imaging solution to investigate the effect of the salt concentration on the hydration structures.

\section{ACKNOWLEDGMENTS}

This work was supported by Grant-in-Aids for Scientific Research from the Ministry of Education, Culture, Sports, Science, and Technology of Japan, SENTAN Program of the Japan Science and Technology Agency, and Global COE Program of the Japanese Society for the Promotion of Science. T.I. is grateful to Professor A. Kovalenko and Professor F. Hirata for providing him with the program code for the 3D-RISM calculation, which was developed in their earlier studies.

${ }^{1}$ D. P. Woodruff, The Solid-Liquid Interface (Cambridge University Press, Cambridge, 1973).

${ }^{2}$ A. J. Bard and L. R. Faulkner, Electrochemical Methods: Fundamentals and Applications, 2nd ed. (John Wiley \& Sons, New York, 2000).

${ }^{3}$ H.-J. Butt, K. Graf, and M. Kappl, Physics and Chemistry of Interfaces (Wiley-VCH, Weinheim, 2003).

${ }^{4}$ Biomineralization: Progress in Biology, Molecular Biology and Application, 2nd ed., edited by E. Bäuerlein (Wiley-VCH, Weinheim, 2004).

${ }^{5}$ T. Fukuma, K. Kobayashi, K. Matsushige, and H. Yamada, Appl. Phys. Lett. 87, 034101 (2005).

${ }^{6}$ T. R. Albrecht, P. Grütter, D. Horne, and D. Ruger, J. Appl. Phys. 69, 668 (1991).

${ }^{7}$ Noncontact Atomic Force Microscopy, edited by S. Morita, R. Wiesendanger, and E. Meyer (Springer, Berlin, 2002).

${ }^{8}$ Noncontact Atomic Force Microscopy, edited by S. Morita, F. J. Giessibl, and R. Wiesendanger (Springer, Berlin, 2009), Vol. 2.

${ }^{9}$ M. A. Henderson, Surf. Sci. Rep. 46, 1 (2002).

${ }^{10}$ M. Chaplin, Nat. Rev. Mol. Cell Biol. 7, 861 (2006).

${ }^{11}$ J. P. Cleveland, T. E. Schäffer, and P. K. Hansma, Phys. Rev. B 52, R8692 (1995).

${ }^{12}$ S. P. Jarvis, T. Uchihashi, T. Ishida, Y. Nakayama, and H. Tokumoto, J. Phys. Chem. B 104, 6091 (2000).

${ }^{13}$ S. Jeffery, P. M. Hoffmann, J. B. Pethica, C. Ramanujan, H. Özgür, and A. Oral, Phys. Rev. B 70, 054114 (2004).

${ }^{14}$ D. Kiracofe and A. Raman, Phys. Rev. B 86, 205405 (2012).

${ }^{15}$ K. Kimura, S. Ido, N. Oyabu, K. Kobayashi, Y. Hirata, T. Imai, and H. Yamada, J. Chem. Phys. 132, 194705 (2010).

${ }^{16}$ K. Suzuki, N. Oyabu, K. Kobayashi, K. Matsushige, and H. Yamada, Appl. Phys. Express 4, 125102 (2011).

${ }^{17}$ K. Tagami and M. Tsukada, e-J. Surf. Sci. Nanotechnol. 4, 294 (2006).

${ }^{18}$ M. Tsukada, N. Watanabe, M. Harada, and K. Tagami, J. Vac. Sci. Technol. B 28, C4C1 (2010).

${ }^{19}$ M. Watkins and A. L. Shluger, Phys. Rev. Lett. 105, 196101 (2010).

${ }^{20}$ D. Argyris, P. D. Ashby, and A. Striolo, ACS Nano 5, 2215 (2011).

${ }^{21}$ T. Fukuma, Y. Ueda, S. Yoshioka, and H. Asakawa, Phys. Rev. Lett. 104, 016101 (2010). 
${ }^{22}$ A. Labuda, K. Kobayashi, D. Kiracofe, K. Suzuki, P. H. Grütter, and H. Yamada, AIP Advances 1, 022136 (2011).

${ }^{23}$ K. Kobayashi, H. Yamada, H. Itoh, T. Horiuchi, and K. Matsushige, Rev. Sci. Instrum. 72, 4383 (2001).

${ }^{24}$ T. Fukuma, M. Kimura, K. Kobayashi, K. Matsushige, and H. Yamada, Rev. Sci. Instrum. 76, 053704 (2005).

${ }^{25}$ D. Sawada, Y. Sugimoto, K. Morita, M. Abe, and S. Morita, Appl. Phys. Lett. 94, 173117 (2009).

${ }^{26}$ K. Momma and F. Izumi, J. Appl. Crystallogr. 44, 1272 (2011).
${ }^{27}$ A. Labuda, K. Kobayashi, K. Suzuki, H. Yamada, and P. Grütter, Phys. Rev. Lett. 110, 066102 (2013).

${ }^{28}$ J. E. Sader and S. P. Jarvis, Appl. Phys. Lett. 84, 1801 (2004).

${ }^{29}$ L. Cheng, P. Fenter, K. L. Nagy, M. L. Schlegel, and N. C. Sturchio, Phys. Rev. Lett. 87, 156103 (2001).

${ }^{30}$ S. H. Park and G. Sposito, Phys. Rev. Lett. 89, 085501 (2002).

${ }^{31}$ Y. Leng and P. T. Cummings, J. Chem. Phys. 124, 074711 (2006).

${ }^{32}$ See supplementary material at http://dx.doi.org/10.1063/1.4803742 for the two movie files. 